\section{Signal combining in LINC amplifier using Alamouti codes}

\section{S. Ali, B. Adebisi, G. Markarian and E. Arikan}

The linear amplification with nonlinear components (LINC) amplification method is discussed. A novel way of combing the equi-magnitude signals is presented. This method uses the space time $2 \times 1$ Alamout code for combining the signal at the receiver. By so doing, the combiner power loss and isolation problems inherent in this technique can be avoided.

Introduction: To address the insatiable demand for high data-rate, various wireless broadband standards have adopted bandwidth efficient modulation schemes such as OFDM in the physical layer $[1,2]$. One of the drawbacks of such multi-carrier schemes is that they suffer from the high peak-to-average power ratio (PAPR) problem. This means that the peak(s) are quite far from the average power. To avoid BER degradation, reduction in efficiency and out of band spectral emissions as a result of the high PAPR, designers usually employ linear amplifiers. The use of linear amplifiers is a costly solution. One of the ways to circumvent this is to use nonlinear amplifiers with a modified signal that has a constant envelope. It is well known that a constant envelope signal can be amplified using a nonlinear device [3]. In this amplification process, a varying envelope signal is split into two constant envelope signals. These components are then amplified individually using nonlinear amplifiers. The outputs of the two amplifiers are subsequently combined to generate a composite amplified signal. This method is known as linear amplification using nonlinear components - referred to as LINC in the literature [4-8]. The main challenge of this technique is the combining of the two amplified signals. One the issues is to design a linear combiner while maintaining high isolation between the output of the two amplifiers [9]. For this reason, the area is still under considerable research.

To get around this challenge, Abdelaal [10] introduced the concept of combining these high-power signals on air, such that the receiver receives the combination of the two signals of LINC. He presented the design and simulation of a $2 \times 1$ LINC amplifier using two antennas at the transmitter. The two transmit antennas are placed close enough such that the channels for each of them could be assumed the same. Thus at the receiving antenna, a sum of the signals is received. This technique provides very good isolation; however, there is a constraint of placing the antennas very close to each other. Therefore practical implementation of such a technique is limited to applications where closely spaced antennas can be tolerated.

This Letter introduces the use of $2 \times 1$ Alamouti space time block codes (STBC) in an LINC based amplification method. This scheme uses a diversity combiner at the receiver to reconstruct the transmitted signal. This is a time multiplexed technique, which can relax the antenna placement constraint in [7]. The spacing can be as much as permitted in the MIMO techniques. However, in this technique the channel is considered to be known at the receiver. Moreover the channel for the two symbol intervals is considered to be unchanged [11]. The complete setup is presented in Fig. 1.

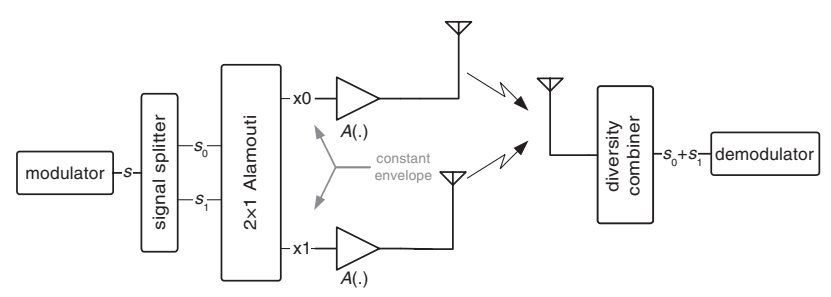

Fig. 1 Split approach for nonlinearity mitigation

Let $S$ be a complex varying envelope baseband signal, which can be represented as

$$
S=a(t)
$$

where $a(t)$ is the complex valued signal. The signal $S$ at any particular instance can be split into two complex and constant magnitude signals $s_{0}$ and $s_{1}$, such that the sum of the two gives back the original signal
$S$. This splitting is represented as

$$
S=s_{0}+s_{1}
$$

This process is illustrated in Figs. 2 and 3 with two different scenarios. It can be seen from the two Figures that, although the magnitude of the input signal is changing, the magnitude of the two split components remains the same. These two constant magnitude components can be amplified through the nonlinear amplifiers and combined again to create a linearised amplified signal.

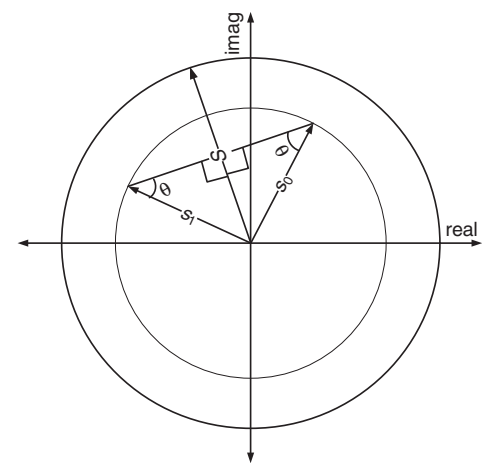

Fig. 2 Splitting of vector into two constant magnitude vectors

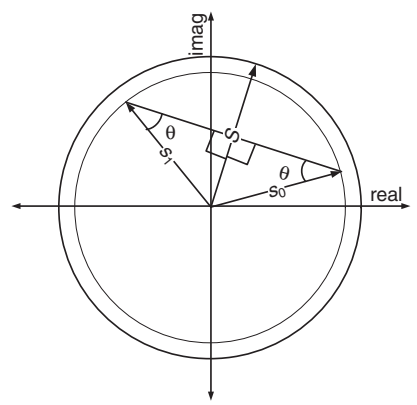

Fig. 3 Splitting of vector into two constant magnitude vectors

If $V_{0}$ is the magnitude of the split components, and $\theta(t)$ is the angle as shown in Figs. 2 and 3 , then $\theta(t)$ is given as

$$
\begin{aligned}
\theta(t) & =\sin ^{-1}\left(\frac{S}{2 s_{1}}\right) \\
& =\sin ^{-1}\left(\frac{a(t)}{2 V_{0}}\right)
\end{aligned}
$$

and the vector $S$ becomes

$$
S=a(t)=2 V_{0}[\sin (\theta(t))]
$$

Thus from trigonometric identity, the two equi-magnitude baseband complex signals are given as

$$
\begin{aligned}
& s_{0}=V_{0}[-\cos (\theta(t))+i \sin (\theta(t))] \\
& s_{1}=V_{0}[\cos (\theta(t))+i \sin (\theta(t))]
\end{aligned}
$$

Their equivalent conjugates are

$$
\begin{aligned}
& s_{0}^{*}=V_{0}[-\cos (\theta(t))+i \sin (\theta(t))] \\
& s_{1}^{*}=V_{0}[\cos (\theta(t))+i \sin (\theta(t))]
\end{aligned}
$$

By applying Alamouti code $s_{0}$ and $s_{1}$ can be transmitted such that the signals at the receiver become

$$
\begin{aligned}
& r_{0}=h_{0} s_{0}+h_{1} s_{1}+n_{0} \\
& r_{1}=-h_{0} s_{1}^{*}+h_{1} s_{0}^{*}+n_{1}
\end{aligned}
$$

where $h_{0}$ and $h_{1}$ are the complex channel gains, and $n_{0}$ and $n_{1}$ are the noise sources in the channels. The estimate of the symbols at the receiver can be obtained using diversity combining as

$$
\begin{aligned}
& \tilde{s}_{0}=h_{0}^{*} r_{0}+h_{1} r_{1}^{*} \\
& \tilde{s}_{1}=h_{1}^{*} r_{0}-h_{0} r_{1}^{*}
\end{aligned}
$$




$$
\begin{aligned}
\tilde{s}_{0} & =h_{0}\left(h_{0} s_{0}+h_{1} s_{1}+n_{0}\right)^{*}+h_{1}^{*}\left(-h_{0} s_{1}^{*}+h_{1} s_{0}^{*}+n_{1}\right) \\
& =\left(\left|h_{0}\right|^{2}+\left|h_{1}\right|^{2}\right) s_{0}+h_{0} n_{0}^{*}+h_{1}^{*} n_{1} \\
\tilde{s}_{1} & =h_{1}\left(h_{0} s_{0}+h_{1} s_{1}+n_{0}\right)^{*}-h_{0}^{*}\left(-h_{0} s_{1}^{*}+h_{1} s_{0}^{*}+n_{1}\right) \\
& =\left(\left|h_{0}\right|^{2}+\left|h_{1}\right|^{2}\right) s_{1}+h_{1} n_{0}^{*}+h_{0}^{*} n_{1}
\end{aligned}
$$

The estimated symbols are then summed together at the receiver to generate the composite signal. This summation is represented as

$$
\begin{aligned}
S= & \hat{s}_{0}+\hat{s}_{1} \\
= & \left(\left|h_{0}\right|^{2}+\left|h_{1}\right|^{2}\right) s_{0}+h_{0} n_{0}^{*}+h_{1}^{*} n_{1} \\
& +\left(\left|h_{0}\right|^{2}+\left|h_{1}\right|^{2}\right) s_{1}+h_{1} n_{0}^{*}+h_{0}^{*} n_{1} \\
= & \left(\left|h_{0}\right|^{2}+\left|h_{1}\right|^{2}\right)\left(s_{0}+s_{1}\right)+h_{0} n_{0}^{*}+h_{1}^{*} n_{1}+h_{1} n_{0}^{*}+h_{0}^{*} n_{1} \\
= & \left(\left|h_{0}\right|^{2}+\left|h_{1}\right|^{2}\right)\left(s_{0}+s_{1}\right)+\left(h_{0}+h_{1}\right) n_{0}^{*}+\left(h_{1}^{*} h_{0}^{*}\right) n_{1}
\end{aligned}
$$

Using (5) and (11) we get

$$
\begin{aligned}
S= & \hat{s}_{0}+\hat{s}_{1} \\
= & \left(\left|h_{0}\right|^{2}+\left|h_{1}\right|^{2}\right)\left(V_{0}[-\cos (\theta(t))+i \sin (\theta(t))]\right. \\
& \left.+V_{0}[\cos (\theta(t))+i \sin (\theta(t))]\right) \\
& +\left(h_{0}+h_{1}\right) n_{0}^{*}+\left(h_{1}^{*}+h_{0}^{*}\right) n_{1} \\
= & \left(\left|h_{0}\right|^{2}+\left|h_{1}\right|^{2}\right)\left(2 V_{0} \sin (\theta(t)) i\right)+\left(h_{0}+h_{1}\right) n_{0}^{*}+\left(h_{1}^{*}+h_{0}^{*}\right) n_{1}
\end{aligned}
$$

Now using (12) with (11) we get the following:

$$
\begin{aligned}
S & =\hat{s}_{0}+\hat{s}_{1} \\
& =\left(\left|h_{0}\right|^{2}+\left|h_{1}\right|^{2}\right)\left(2 V_{0} \sin (\theta(t)) i\right)+\left(h_{0}+h_{1}\right) n_{0}^{*}+\left(h_{1}^{*}+h_{0}^{*}\right) n_{1} \\
& =\left(\left|h_{0}\right|^{2}+\left|h_{1}\right|^{2}\right)\left(2 V_{0} \sin \left(\sin ^{-1}\left(\frac{a(t)}{2 V_{0}}\right)\right) i\right)+\left(h_{0}+h_{1}\right) n_{0}^{*}+\left(h_{1}^{*}+h_{0}^{*}\right) n_{1} \\
& =\overbrace{\left(\left|h_{0}\right|^{2}+\left|h_{1}\right|^{2}\right)(a(t))}^{\text {recovered }}+\left(h_{0}+h_{1}\right) n_{0}^{*}+\left(h_{1}^{*}+h_{0}^{*}\right) n_{1}
\end{aligned}
$$

Conclusions: In a LINC amplifier the overall performance of the system relies heavily on the signal combiner placed at the outputs of the amplifiers. The major issue with such a combiner is the isolation between the two amplifiers. We present a novel signal combining technique that resolves this issue. The combining is done at the receiver using $2 \times 1$ Alamouti STBC codes. In this way a complete isolation can be achieved between the two amplifying chains. The concept has been presented using mathematical derivation. Overall, this technique promises the mitigation of the isolation problem, elimination of combiner power loss and relaxation in antenna spacing requirements.

Acknowledgments: This work was supported in part by the EU FP7 project WiMAGIC under grant agreement no. 215167. The authors acknowledge the support provided by RINICOM Ltd.

(C) The Institution of Engineering and Technology 2010

15 April 2010

doi: $10.1049 / \mathrm{el} .2010 .1015$

S. Ali, B. Adebisi, G. Markarian and E. Arikan (Communications Systems, Lancaster University, Room B28, Infolab21, Lancaster LA14 WA, United Kingdom)

E-mail: a.saqib@lancaster.ac.uk

\section{References}

1 Eklund, C., et al.: 'IEEE standard 802.16: a technical overview of the WirelessMAN TM air interface for broadband wireless access', IEEE Commun. Mag., 2002, 40, pp. 98-107

2 Seidel, E.: 'Progress on "LTE Advanced" - the new 4G standard', White Paper, Nomor Research, 2008

3 Liang, C., et al.: 'Nonlinear amplifier effects in communications systems', IEEE Trans. Microw. Theory Tech., 1999, 47, pp. 1461-1466

4 Cox, D.: 'Linear amplification with nonlinear components', IEEE Trans. Commun., 1974, (legacy, pre-1988) 22, pp. 1942-1945

5 Casadevall, F., and Valdovinos, A.: 'Performance analysis of QAM modulations applied to the LINC transmitter', IEEE Trans. Veh. Technol., 1993, 42, pp. 399-406

6 Choffrut, A., et al.: 'Traveling wave tube-based LINC transmitters', IEEE Trans. Electron Devices, 2003, 50, pp. 1405-1407

7 Helaoui, M., et al.: 'A new mode-multiplexing LINC architecture to boost the efficiency of WiMAX up-link transmitters', IEEE Trans. Microw. Theory Tech., 2007, 55, pp. 248-253

8 Stengel, B., and Eisenstadt, W.: 'LINC power amplifier combiner method efficiency optimization', IEEE Trans. Veh. Technol., 2000, 49, pp. 229-234

9 Poitau, G., et al.: 'Experimental characterization of LINC outphasing combiners' efficiency and linearity'. IEEE Radio and Wireless Conf., Atlanta, GA, USA, 2004, pp. 87-90

10 Abdelaal, M.M.: 'LINC based amplifier architectures for power efficient wireless transmitters', PhD Thesis, 2009, Ecole Polytechnique (Montreal, Canada)

11 Alamouti, S.M.: 'A simple transmit diversity technique for wireless communications', IEEE J. Sel. Areas Commun., 1998, 16, pp. $1451-1458$ 